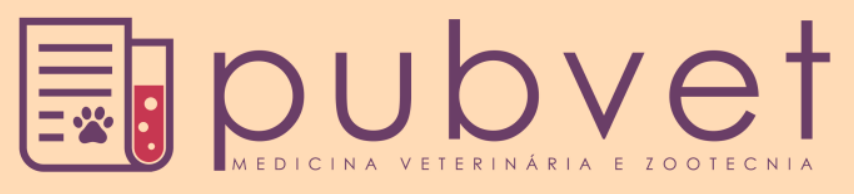

https://doi.org/10.22256/pubvet.v12n2a26.1-4

\title{
Meningioma canino: Relato de caso
}

\section{Lourival Barros de Sousa Brito Pereira ${ }^{*}$, Homero Firmo Pessoa², Gabriela de Paiva Sousa $^{1}$, Lucilo Bioni da Fonsêca Filho ${ }^{\bullet 3}$, Ana Luiza Neves Guimaraes Bessa ${ }^{\bullet}{ }^{4}$, Priscilla Virginio de Albuquerque ${ }^{\bullet}$, Silvia Fernanda de Alcantara ${ }^{\bullet}$, Júlio Cézar dos Santos Nascimento ${ }^{\bullet}$, Marleyne José Afonso Accioly Lins Amorim ${ }^{\bullet}$}

${ }^{I}$ Discente do curso de Medicina Veterinária, Universidade Maurício de Nassau, Recife-PE Brasil.

${ }^{2}$ Médico Veterinário especializado em oncologia Recife-PE Brasil.

${ }^{3}$ Discente do curso de Medicina Veterinária em Universidade Federal Rural de Pernambuco, Recife-PE Brasil. E-mail: lucilofilho@ gmail.com

${ }^{4}$ Professor do Centro Universitário Maurício de Nassau, Curso de Medicina Veterinária, Recife-PE Brasil.

${ }^{5}$ Departamento de Morfologia e Fisiologia Animal, Universidade Federal Rural de Pernambuco Recife-PE Brasil. E-mail:

${ }^{6}$ Professor Associado em Universidade Federal Rural de Pernambuco, Recife-PE Brasil. E-mail: marleyneamorim@gmail.com

*Autor para correspondência, E-mail: lorinho2013.1@ hotmail.com

RESUMO. O meningioma é a neoplasia intracraniana mais comum em cães na clínica veterinária, em maior parte dos casos possui caráter benigno, além disso, pode vir a surgir em qualquer uma das três meninges que revestem e protegem o sistema nervoso central. $\mathrm{O}$ principal sinal clínico do meningioma é a convulsão, porém alteração de comportamento vem sendo relatada com frequência na literatura. A lomustina por ser altamente solúvel em lipídeos, atinge elevadas concentrações no fluido cérebro-espinhoso permitindo que a mesma seja usada como protocolo quimioterápico para pacientes com meningioma. Objetivou-se com esse trabalho, relatar um caso de meningioma intracraniano em um cão de raça poodle com dez anos de idade destacando sua evolução clínica, diagnóstico presuntivo e conduta terapêutica.

Palavras chave: convulsão, lomustina, neoplasia intracraniana

\section{Canine meningioma: Case report}

ABSTRACT. Meningioma is the most common intracranial neoplasia in dogs in the veterinary clinic, in most cases it has a benign nature, and may arise in any of the three meninges that cover and protect the central nervous system. The main clinical sign of meningioma is seizure, but behavioral alterations have been reported frequently in the literature. Lomustine, because it is highly soluble in lipids, reaches high concentrations in the brain-spinal fluid allowing it to be used as a chemotherapeutic protocol for patients with meningioma. The objective of this work was to report a case of intracranial meningioma in a poodle dog at the age of ten, highlighting its clinical evolution, presumptive diagnosis and therapeutic behavior.

Keywords: convulsion, lomustine, intracranial neoplasia

\section{Meningioma canino: Reporte de un caso}

RESUMEN. El meningioma es la neoplasia intracraneal más común en perros en la clínica veterinaria, en la mayoría de los casos tiene carácter benigno, además, puede surgir en cualquiera de las tres meninges que revisten y protegen el sistema nervioso central. La principal señal clínica del meningioma es la convulsión, pero alteración de comportamiento viene siendo reportada con frecuencia en la literatura. La lomustina por ser altamente soluble en lípidos, alcanza altas concentraciones en el fluido cerebro-espinoso permitiendo 
que la misma sea usada como protocolo de quimioterapia para pacientes con meningioma. Se objetivó con ese trabajo, relatar un caso de meningioma intracraneal en un perro de raza Poodle con diez años de edad destacando su evolución clínica, diagnóstico presuntivo y conducta terapéutica.

Palabras clave: convulsiones, lomustina, neoplasia intracraneal

\section{Introdução}

Com o avanço tecnológico na Medicina Veterinária, doenças que eram incomuns, passaram a ser diagnosticadas com maior frequência, como é o caso de neoplasias intracranianas (Carvalho et al., 2017).

Os principais tumores encefálicos primários em cães são os meningiomas, gliomas e tumores neuroepiteliais (Silva et al., 2014). O meningioma é o tumor do sistema nervoso central mais comum no cão, podendo ter origem em qualquer uma das três meninges (Teixeira, 2015) e ocorre mais frequentemente em animais entre 12 a 14 anos de idade (Silva et al., 2014).

Apesar de o meningioma canino ter um caráter infiltrativo maior que o de gato (Daleck et al., 2016) . É considerada uma neoplasia benigna, de crescimento lento que compromete as estruturas neurais adjacentes (Babicsak et al., 2011). O lobo frontal é a região no cérebro mais acometida pelo meningioma (Silva et al., 2014).

A etiologia para ocorrência das neoplasias intracranianas não são totalmente esclarecidas; porém a dieta, o ambiente, trauma craniano, fatores genéticos, imunológicos, químicos e virais podem predispor ao aparecimento (Marcasso et al., 2015). Teixeira (2015) em seu estudo relata que as raças de maior predisposição para o meningioma incluem o pastor alemão, Colie, Golden Retriever, Labrador Retriever e Boxer.

Geralmente, animais portadores de neoplasia intracranianos apresentam um aparecimento gradual dos sinais clínicos (Marcasso et al., 2015, Carvalho et al., 2017). O principal sinal clínico encontrado em um quadro de menigioma são as convulsões focais ou generalizadas (Silva et al., 2014, Marcasso et al., 2015), edema, hidrocefalia e hemorragia podem ocorrer na forma secundária a doença. Outros sinais clínicos que podem estar associadas às neoplasias intracranianas são síndrome vestibular, cegueira, dor em região cervical, anisocoria, tremores de cabeça e regurgitação.

Estudos recentes relatam alteração de consciência como o sinal clínico de maior ocorrência (Marcasso et al., 2015). Contudo os sinais clínicos vão depender da lesão do tecido neural adjacente ao tumor e do edema cerebral associado (Teixeira, 2015).

Segundo Silva et al. (2014) há uma relação entre malignidade de meningioma e a expressão de receptores para progesterona e estrógeno, sendo elevada a expressão de receptores para progesterona em meningiomas benignos e baixa ou nenhuma expressão de receptores para progesterona em meningiomas malignos.

Para se ter o diagnóstico presuntivo, a tomografia computadorizada e ressonância magnética são exames de imagem mais utilizados (Silva et al., 2014) sendo esta associada com o histórico, a raça e a idade do animal e os sinais neurológicos apresentados e sua progressão (Carvalho et al., 2017). A tomografia computadorizada é menos sensível do que a ressonância magnética. Suas vantagens incluem excelentes visualizações de lesões ósseas, calcificação e hemorragias agudas. A ressonância magnética apresenta disponibilidade limitada, requer anestesia geral prolongada e custo elevada; porém é considerado o melhor método neurodiagnóstico (Daleck et al., 2016) .

A radiografia simples é muito limitada pelo fato de que a maioria das neoplasias intracranianas envolve meninges ou o parênquima encefálico não sendo possível a visualização neste exame (Daleck et al., 2016) . Para diagnóstico definitivo necessita-se de exame histopatológico (Silva et al., 2014) e mesmo que não possa diagnosticar ante mortem, é importante que o clínico/médico veterinário obtenha um diagnostico final no pos mortem.

O objetivo do tratamento definitivo é dar qualidade de vida ao animal pelo maior tempo possível e isso é possível por meio da cirurgia, radioterapia e quimioterapia (Daleck et al., 2016). Geralmente a cirurgia é a terapêutica que oferece maior chance cura. Os glicocorticoides auxiliam no tratamento pois reduz o edema peritumoral (Marcasso et al., 2015). Os anticonvulsivantes são utilizados para reduzir as manifestações clínicas (Silva et al., 2014). 
A lomustina é um quimioterápico que atinge rapidamente elevadas concentrações no fluido cérebro-espinhoso o que permite que seja empregado em neoplasias do sistema nervoso central (Rodaski, 2008); porém existe pouca informação quanto a eficácia da quimioterapia para neoplasias intracraniana em cães e gatos (Daleck et al., 2016).

\section{Relato de caso}

Foi encaminhado a uma clínica veterinária um cão de raça Poodle, fêmea, castrada com dez anos de idade, pesando aproximadamente $9 \mathrm{~kg}$ para uma consulta oncológica a respeito de dois tumores de mama. Após a realização da cirurgia para retirada dos tumores de mama, foi feito exame histopatológico e os mesmos foram classificados como carcinoma mamário misto grau um e carcinoma mamário papilífero grau dois. Animal estava bebendo e comendo normalmente, sem alterações de micção e defecação com protocolo vacinal em dia.

Todavia, a tutora relata episódios de convulsões frequentes, vocalização sem causa aparente, com evolução gradativa, sem histórico de trauma, já havia feito o uso de corticoides e fenobarbital melhorando os sinais clínicos; porém com recidivas. No exame físico o animal não apresentava alterações neurológicas.

Visto a possibilidade de metástase, foram solicitados radiografia torácica, ultrassom abdominal e tomografia computadorizada para avaliar a região da cabeça, além do hemograma e bioquímica renal e hepático. Pelo quadro convulsivo foi prescrito brometo de potássio e corticosteroide $5 \mathrm{mg}$ dia.
No exame de tomografia computadorizada (Figura 1), nos mostrou uma massa em lobo frontal direito com formato amorfo, de comprimento $1,94 \mathrm{~cm}$, altura $3,15 \mathrm{~cm}$ e largura de $1,93 \mathrm{~cm}$, promovendo desvio da linha média para a esquerda. Isso sugere meningioma com base nas características da lesão e relata que a possibilidade de ser uma lesão metastática é baixa. Visto também sinais de compressão do sistema ventricular direito e edema vasogênico de grande extensão. Os demais exames não apresentavam alterações relevantes.

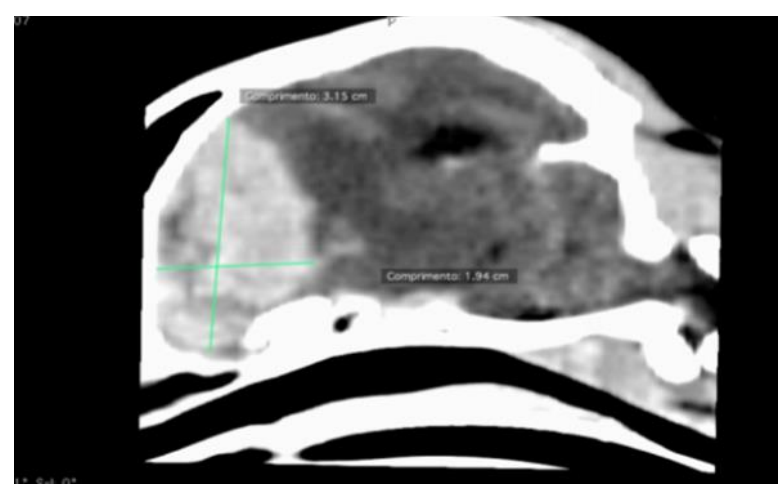

Figura 1. Primeiro exame tomográfico. Neoplasia com 3,15 x $1,94 \mathrm{~cm}$ de comprimento.

Dois dias após o diagnóstico presuntivo de meningioma, foram iniciadas as sessões de quimioterapia com lomustina na dose de $22 \mathrm{mg}$ a cada vinte e um dias associado com um glicocorticoide diariamente. Após sete meses, totalizando sete sessões com lomustina, o paciente realizou outra tomografia computadorizada e quando comparado com o exame anterior observase redução aproximada de $20 \%$ do tumor (igura 2).

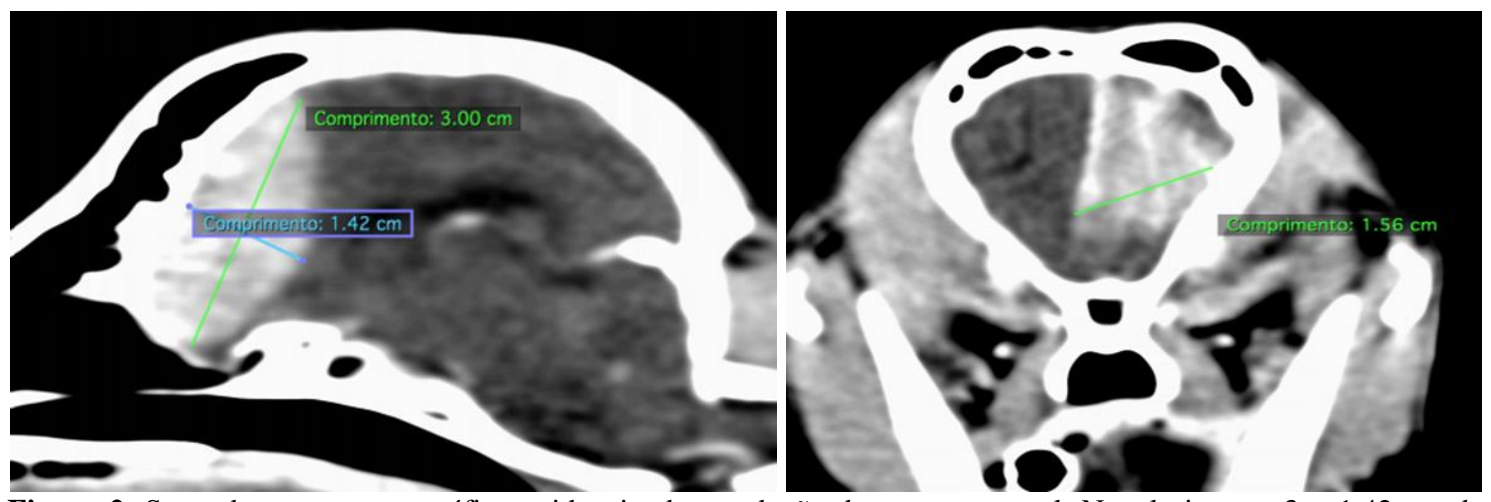

Figura 2. Segundo exame tomográfico evidenciando a redução da massa tumoral. Neoplasia com 3 x $1,42 \mathrm{~cm}$ de comprimento e 1,56 de largura.

Dentre os sete meses de terapia, a proprietária relata que houve melhora significativa dos sinais clínicos. Nos exames hematológicos feitos antes de cada sessão de quimioterapia era notório as alterações na fosfatase alcalina; porém sempre a controlando permitindo que o médico veterinário 
optasse por dar continuidade ao tratamento, alterando o intervalo entre cada sessão de quimioterapia, passando de vinte e um dias para trinta e cinco dias, associado ao corticosteroide diariamente. Desde o diagnóstico presuntivo até o presente momento, fazem doze meses de tratamento com acompanhamento hematológico mensalmente e o paciente continua sem sintomas clínicos.

\section{Discussão}

A cadelinha tem 10 anos, pouco abaixo da estimativa relatada por Silva et al. (2014) que relatam que ocorre com mais frequência em animais de 12 a 14 anos e condizente com a relatada por Marcasso et al. (2015) de 10 anos.

A predisposição relatada por Carvalho et al. (2017) que foram cães de raça Boxer, Golden Retriever, Doberman, Scottish Terrier e Old Inglês Sheepdog, por Silva et al. (2014) cães de grande porte e raças dolicocefálicas não condiz com a raça do animal do relato de caso.

A alteração de comportamento e quadros convulsivos apresentados no caso corrobora com o que é relatado pela literatura como sinais clínicos comumente vistos pelos proprietários.

O diagnóstico presuntivo de meningioma foi com base na anamnese, sendo um animal na faixa etária, sinal neurológico com evolução do sinal e a tomografia como exame complementar.

O tumor localizava-se no lobo frontal, região de maior ocorrência desta neoplasia (Silva et al., 2014).

O animal estava livre de sinais clínicos da doença há mais ou menos 370 dias com tratamento com glicocorticoides e lomustina. Uma sobrevida superior a relatada por Silva et al. (2014) que é de 3 meses para animais que receberam apenas tratamento de suporte. Resultado satisfatório com o uso da lomustina e o glicocorticoide como protocolo terapêutico no meningioma.

Alterações hematológicas foram vistos na fosfatase alcalina que oscilou de 200 a $2.000 \mathrm{U} / \mathrm{I}$ durante o protocolo terapêutico.

\section{Conclusão}

Neoplasias intracranianas devem estar incluídas no diagnostico diferencial para animais idosos. A lomustina associada a um glicocorticoide mostrou ser eficaz na terapia de neoplasias intracranianas, sendo o acompanhamento hematológico periódico indispensável pelo fato do grande potencial mielotóxico e hepatotóxico que a lomustina apresenta.

\section{Referências Bibliográficas}

Babicsak, V. R., Zardo, K. M., Santos, D. R., Silva, L. C., Vasconcelos Machado, V. M. \& Vulcano, L. C. 2011. Aspectos tomográficos de tumores cerebrais primários em cães e gatos. Veterinária e Zootecnia, 18, 531-541.

Carvalho, J. R. G., Carvalho, V. C. H., Bastos, I. P. B., Carvalho, T. F. L., Costa, T. S. \& Fernandes, J. I. 2017. Meningioma intracraniano canino: Relato de caso. Brazilian Journal of Veterinary Medicine, 38, 1-7.

Daleck, C. R., Fonseca, C. S. \& Canola, J. C. 2016. Oncologia em cães e gatos. Roca, Rio de Janeiro.

Marcasso, R. A., Moreira, J. R., Valentim, L. G., Arias, M. V. B. \& Bracarense, A. P. F. R. L. 2015. Meningiomas em cães: aspectos clínicos, histopatológicos e imuno-histoquímicos. Pesquisa Veterinária Brasileira, 35, 844-852.

Rodaski, S. 2008. Quimioterapia antineoplásica em cães e gatos. MedVet Livros, São Paulo.

Silva, P. D. G., Nardotto, J. R. B., Filgueiras, R. d. R. \& Mortari, A. C. 2014. Neoplasias intracranianas primárias em cães. Revista Científica de Medicina Veterinária, 12, 1188211882.

Teixeira, V. S. G. 2015. Meningiomas do sistema nervoso central em cães. Departamento de Veterinária. Universidade Trás-os-Montes e Alto Douro, Vila real.

Article History:

Received 18 October 2017

Accepted 25 October 2017

Available online 8 January 2018

License information: This is an open-access article distributed under the terms of the Creative Commons Attribution License 4.0, which permits unrestricted use, distribution, and reproduction in any medium, provided the original work is properly cited. 